\title{
Arte público y políticas culturales en el \\ posconflicto: potencias, retos y límites
}

\section{Artículo de investigación}

\section{Adriana Roque Romero}

Investigadora independiente, Bogotá, Colombia aroque88@gmail.com

Recibido: 22 de julio de 2017

Aprobado: 20 de diciembre de 2017

Como citar este artículo: Roque Romero, Adriana (2018). Arte público y políticas culturales en el postconflicto: potencias, retos y límites.Calle 14: revista de investigación en el campo del arte 13 (24) pp. 360-374. DOI: https://doi.org/10.14483/21450706.13531

Este artículo es producto de la investigación teórica emprendida en el proyecto de grado de maestría y de vinculación al proceso de formulación de políticas culturales desde la Secretaría Distrital de Cultura, Recreación y Deporte de la ciudad de Bogotá, durante el año 2015. También ha sido alimentada por varios talleres y charlas dadas por la autora en el contexto académico, relacionadas con estudios de conflicto y prácticas artísticas. 
Arte público y políticas culturales en el posconflicto: potencias, retos y límites

\begin{abstract}
Resumen
En el presente artículo se aborda la pregunta sobre el lugar del arte en la coyuntura de la transición al posconflicto colombiano, en relación con las políticas culturales. Desde una aproximación teórica filosófica, se aborda las prácticas artísticas desde los planteamientos de régimen estético, política y reparto de lo sensible, del filósofo francés Jacques Rancière, para entender la potencia propia de las prácticas artísticas en el posconflicto como una potencia del disenso, de la apertura de espacios de novedad donde las narrativas, heridas y la constitución misma de la comunidad entran en cuestión. Para entender el lugar de las políticas culturales en este contexto, se plantea la posibilidad de pensar la importancia del arte público, con sus virtudes y peligros. Finalmente, se introduce la necesidad de repensar las políticas culturales para permitir expresiones y manifestaciones artísticas propias de una situación de transición y de posconflicto.
\end{abstract}

\title{
Palabras claves
}

Arte público, Colombia, disenso, Jacques Rancière, políticas culturales, posconflicto.

Public Art and cultural policies in the post-conflict era: powers, challenges and limits

\begin{abstract}
In this article I approach the issue of the place of artistic practices in the political juncture of the transition to a post-conflict era in Colombia, in relation to cultural policies. From a philosophical perspective, I approach artistic practices based on Jacques Rancière's concepts of aesthetic regime, politics and distribution of the sensible. This will allow us to understand the potential of artistic practices in a post-conflict scenario as a source of dissent, of the creation of novel spaces where narratives, wounds and the constitution of community itself come into question. To understand the place of artistic practices, I propose to revise the importance of public art, taking into account the potentiality and dangers of this form of art. Finally, the necessity of new cultural policies for the post-conflict era is proposed, in order to open the space for artistic manifestations and interventions proper to this specific political situation.
\end{abstract}

\section{Keywords}

Colombia, cultural policies, dissent, Jacques Rancière, post-conflict, public art.

\section{Politiques publiques d'art et de culture dans l'après-conflit :Pouvoirs, défis et limites}

\section{Résumé}

Dans le présent article, la question de la place de l'art dans la conjoncture de la transition au post-conflit colombien, en relation avec les politiques culturelles, est abordée. Partant d'une approche théorique philosophique, les pratiques artistiques sont abordées à partir des concepts du régime esthétique, de la politique et de la distribution du sensible du philosophe français Jacques Rancière, pour comprendre le pouvoir des pratiques artistiques dans le post-conflit comme un source de dissidence, et pour l'ouverture d'espaces de nouveauté où les récits, les blessures et la constitution même de la communauté sont remis en question. Pour comprendre la place des politiques culturelles dans ce contexte, la possibilité de réfléchir à l'importance de I'art public, avec ses vertus et ses dangers, est évoquée. Enfin, il est nécessaire de repenser les politiques culturelles pour permettre les expressions et les manifestations artistiques propres à une situation de transition et d'après conflit.

\section{Mots clés}

Art public, Colombie, dissidence, Jacques Ranciére, politiques culturelles, post-conflit. 


\section{Resumo}

Neste artigo, é discutida a questão do lugar da arte no contexto da transição para o pós-conflito colombiano, em relação às políticas culturais. A partir de uma abordagem teórica filosófica, as práticas artísticas são abordadas a partir do regime estético, da política e da distribuição do sensível do filósofo francês Jacques Rancière, para compreender o poder das práticas artísticas no pós-conflito como fonte de dissidência, de abertura de espaços de novidade onde as histórias, as feridas e a própria constituição da comunidade são questionadas. Para compreender o lugar das políticas culturais neste contexto, evoca-se a possibilidade de refletir sobre a importância da arte pública, com suas virtudes e seus perigos. Finalmente, é necessário repensar as políticas culturais para permitir expressões e manifestações artísticas de uma situação de transição e pós-conflito.

\section{Palavras chaves}

Arte pública, Colômbia, dissidência, Jacques Ranciére, políticas culturais, pós-conflito.

\section{Rurai kawachispa kausai rurangapa imasapas nukanchi pudiskasina}

\section{Maillallachiska:}

Kawanakumi imam rurangapa kanchi kunaura manda wañachiiikuna manima tiangapa ka allilla kawaspa iullarispa llukanchimi nukanchi familia sina maki kaunakuna suma kawaspa nupasinama katichingapa tukuikuna kawachispa kunaura charami pudinchi allillu kausanga chasallata Nukanchipa allí mukuisitu charami llukanchi.

\section{Rimangapa Ministidukuna:}

Kawachispa tukuikunata, Nukanchipa atun chagta, rurai, Jacques Ranciere, chasa suti kuna, tukuikunamanda, ñugpasinama kawachispa. 
En su poder de descubrir relaciones no visibles dentro de la sociedad,

de emparentar la acción del hombre con sus motivaciones profundas, de revelar mecanismos peculiares de tal o cual comportamiento social, y de arrojar luz sobre el progresivo esclarecimiento de grupos humanos que se desconocen enteramente a sí mismos. En tal caso, la cultura de la resistencia rebasa su finalidad estética, y toca una ética y hasta una epistemología.

Marta Traba, La cultura de la resistencia (p. 142).

\section{Introducción}

Desde que el gobierno hizo públicos en 2012 los diálogos exploratorios con la guerrilla de las FARC-EP que resultarían en la instalación de la mesa de negociaciones en La Habana, una salida política al conflicto interno armado, se comenzó a hablar de lo que se denomina como 'posconflicto'. Este se materializó con la firma (en dos ocasiones) de los acuerdos de paz, su posterior refrendación y los primeros pasos de su implementación. Aunque los procesos de posconflicto no son algo nuevo en el mundo, se puede argumentar que en Colombia nos enfrentamos a una situación completamente nueva. Si bien se han llevado a cabo desmovilizaciones de diversos grupos armados al margen de la ley ${ }^{1}$ en distintos momentos de nuestra historia política, ninguno ha implicado la desmovilización y reinserción en la escala que los diálogos de La Habana han concretado. Estos diálogos no solo implican la desmovilización de un grupo armado, sino una serie de reformas agrarias, políticas, estatales y sociales que muy probablemente tomarán varios años y marcarán una nueva época en la historia de Colombia².

Así, los retos para el Estado, la empresa privada, las universidades, las organizaciones civiles y la ciudadanía en general, serán grandes y complejos.
En este escenario de cambios e incertidumbres institucionales, sociales y políticas, podemos preguntarnos por el papel de las prácticas artísticas en un proceso de posconflicto. Para abordar esta reflexión, debemos plantearnos preguntas sobre el rol, la funcionalidad y la potencia de estas prácticas, tales como: ¿cuál es el lugar de las prácticas artísticas y culturales en el posconflicto? ¿Éste se limita a una función terapéutica, de construcción de memoria, o de denuncia? ¿Hay algún tipo de deber ético de las artes para involucrarse en este proceso? ¿Es necesaria una política pública para prácticas artísticas y culturales en el posconflicto? ¿Si sí, a qué deberían apuntar? ¿Cómo se verían las prácticas artísticas del posconflicto?

Abordar estas preguntas implica una complejidad singular en la medida en que ellas atañen a varios campos disciplinarios tales como: estudios artísticos, políticas públicas culturales y estudios de posconflicto, entre otros. En el presente artículo se proponen algunos conceptos guía teniendo en cuenta las complejidades ya mencionadas, intentando llamar la atención sobre la singularidad del momento que vive Colombia y que se extenderá muy probablemente por varios años.

Así se proponen cuatro secciones. En primer lugar, haré unas breves anotaciones sobre lo que implica para la presente discusión la coyuntura actual de transición a posconflicto en Colombia, teniendo en cuenta particularmente las problemáticas que surgen en este tipo de procesos desde los ámbitos culturales y políticos. En segundo lugar, plantearé el lugar del arte en este contexto como una potencia política de las prácticas artísticas desde la noción de disenso de Jacques Rancière (2012). En tercer lugar, propongo la noción de 'arte público' como posible punto intermedio entre la potencia disensual de las artes y los constreñimientos institucionales que una política cultural del posconflicto puede acarrear, teniendo en cuenta la ambivalencia propia del arte público. Finalmente, se recogerán las conclusiones principales de esta investigación de forma propositiva para interpretar y abordar la coyuntura histórica en que nos encontramos.

\section{La transición histórica}

Para abordar esta discusión, debemos analizar nuestro punto de partida, no solamente teórico sino también histórico y político. A diferencia del clima político posterior al inicio de la implementación de los acuerdos de paz, lo que presenciamos en estos momentos son 
apenas las medidas inmediatas iniciales de la implementación de dichos acuerdos. Es importante tener en cuenta que los procesos de posconflicto pueden durar varios años, incluso décadas. Los primeros años presentan generalmente los mayores índices de fracaso de este tipo de procesos y la reanudación de la guerra (Nasi y Rettberg, 2005, p. 71). Así pues, nos encontramos, desde todas las áreas y disciplinas, iniciando uno de los momentos claves de una transición al posconflicto: es en estos años donde estos procesos se pueden estabilizar y abrir el camino para los cambios de mayor trascendencia y a largo plazo, o pueden fracasar.

Otra característica que debe tenerse en cuenta es que nos encontramos, precisamente, en un momento de transición. Para muchos teóricos de los estudios de conflicto y paz de corte maximalista, los procesos de transición y posconflicto tienen múltiples capas y niveles (Fischer, 2011; Webel y Galtung, 2007; USIP, 2009; Miall, 2004). Si bien hay discrepancias respecto de los énfasis y la importancia de cada nivel, es claro algo: un proceso de posconflicto y de reconciliación no depende únicamente de la implementación de políticas de desarme y de procesos políticos estructurales. Hay también elementos sistémicos, sociales, históricos y discursivos que hacen parte de este proceso, que entrarán en disputa y que necesariamente deberán abordarse y transformarse.

Podemos decir, de forma muy general, que estamos en medio del (pos)conflicto. Estamos en medio del conflicto porque, aún con la firma de los acuerdos derivados de los diálogos de La Habana hay otros conflictos directos que siguen vigentes: con el ELN; con el narcotráfico; con las bandas criminales; con las disidencias de las FARC que alimentan estructuras criminales. Esto, sin mencionar las formas de conflicto estructurales y sistémicas — por naturaleza más complejas - relacionadas con los altos niveles de desigualdad socio-económica, los niveles de pobreza, la necesidad de inversión en el campo y en zonas y departamentos olvidados, la violencia de género, el racismo y la exclusión política. Estos son conflictos que en los años que vienen pueden amilanarse o intensificarse.

En otro sentido, estamos en medio del (pos)conflicto porque, una vez firmados los acuerdos de paz e iniciada su implementación, en efecto este conflicto singular ha finalizado por vía negociada. Así, nos encontramos en un momento temporalmente posterior al conflicto. Pero, en un sentido más amplio, podemos también argumentar que en el posconflicto nos enfrentaremos a la tarea de imaginar una nueva sociedad cuya columna vertebral no sea la de un conflicto interno armado permanente y todo lo que de él se deriva.

Ahora bien, las necesidades culturales y sociales en una situación de transición a posconflicto no sólo atañen a asuntos simbólicos y no se limitan a actos propagandísticos, pues la vida política, el tejido social y cultural, y las narrativas mismas de nación se han visto profundamente transformadas por la guerra y las violencias que han dado forma y lugar a nuestra historia (GMH, 2013, p. 16). Así pues, nos encontramos en una transición que nos exige pensar y entender la realidad de forma distinta. Es una transición que abre el espacio para discutir y disputar viejas injusticias, nuevas narrativas, y otras formas de reconocimiento. Todo esto toca elementos no solo institucionales, sino particularmente sociales y culturales. En este sentido, son asuntos que pueden ser abordados de formas extra-oficiales y extra-académicas. De igual manera, es un momento en que tampoco los actores que abordan estos asuntos tienen que ser los convencionales. No solo se interpela a los políticos, académicos, u organizaciones que históricamente han estado involucradas con el conflicto interno armado y sus implicaciones, sino también a la ciudadanía en general como parte de una nación, cuestionando su lugar y rol (o su ausencia) en este proceso desde su cotidianidad, sus experiencias y sus conocimientos.

En este contexto, considero pertinente plantear que las prácticas artísticas y su carácter inherentemente creativo, tienen la potencia de crear un espacio nuevo en el cual imaginar otras narrativas de nación, nuevas formas de reconocimiento, sea posible.

\section{La potencia disensual del arte}

Puede parecer contradictorio afirmar que para superar un conflicto necesitemos espacios de disenso y no de consenso. Sin embargo, cuando se plantea la perspectiva de posconflicto a largo plazo, como proceso integral, es crucial que las estructuras sociales permitan no solo la tolerancia de la diferencia, sino la inclusión real —simbólica y material— de la diversidad ${ }^{3}$. Una

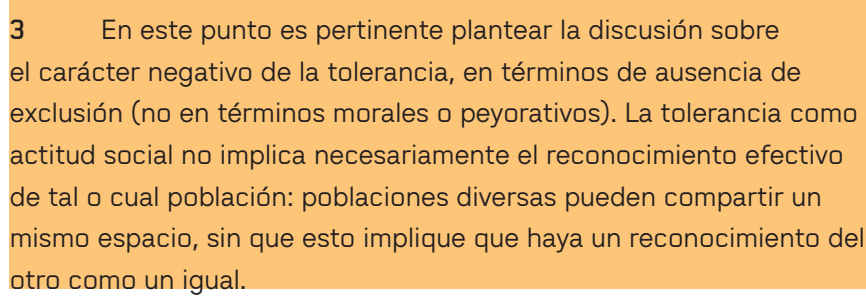


sociedad diversa implica, por definición, la simultaneidad de formas de vida diferentes $y$, en este sentido, de encuentros y choques de esas mismas diferencias. Así, antes que tender a una homogenización social, al establecimiento de status quo político y vital, es importante abrir la posibilidad de la ocurrencia del disenso y la diferencia sin resolución. En efecto, podemos pensar como Estanislao Zuleta, que la posibilidad de la paz radica en la capacidad de la sociedad de acoger estos conflictos y transformarlos, no de aplacarlos de forma autoritaria: "para mí una sociedad mejor es una sociedad capaz de tener mejores conflictos. De reconocerlos y contenerlos. De vivir no a pesar de ellos, sino productiva e inteligentemente en ellos" (2004, pp. 73-74).

Desde esta perspectiva, quiero proponer que en este momento de transición y de posconflicto las prácticas artísticas tienen la potencia de generar esos espacios de disenso, de desacuerdo radical, que permitan imaginar y materializar formas nuevas de habitar, reconocer y disentir. En este sentido, los planteamientos del filósofo francés Jacques Rancière nos permiten entender que esta potencia del arte no es una obligación ética o un deber de las prácticas artísticas. De hecho, como veremos, imponer este tipo de obligaciones o labores éticas neutraliza la potencia real de las prácticas artísticas.

A través de toda su obra, Rancière ha procurado repensar y redefinir dos términos fundamentales para la interpretación contemporánea de las prácticas artísticas: la "estética" y la "política". Estos dos conceptos han sido abordados de múltiples maneras por distintos pensadores, disciplinas y ciencias. En el caso de Rancière, la tarea de redefinir estos conceptos traza la forma en que podemos entender cómo las subjetividades se constituyen, cómo son visibles, y cómo el arte y la política se entrecruzan en las maneras en que enunciamos, entendemos, vemos y simbolizamos dichas subjetividades y las comunidades.

Para Rancière, toda comunidad comporta un "reparto de lo sensible" específico. El reparto de lo sensible se refiere a las formas en que significamos y enmarcamos 'lo común', entendiendo por esto último las formas de visibilidad, identificación y enunciación de diferentes subjetividades, experiencias, objetos y prácticas. Así, más allá de los lineamientos institucionales, una comunidad se compone de singularidades que comparten una experiencia sensible y simbólica común. Es desde esa experiencia cultural y territorial en-común que construyen las formas de dividir y repartir las partes, los lugares, las condiciones materiales y las actividades que componen a la comunidad.

Hay pues un trasfondo sensible que abarca cuerpos, acciones y trabajos, y que de esta forma provee la experiencia común de un sentido, de una forma de entender y significar ${ }^{4}$. Estas formas de enmarcar, entender y repartir lo común no constituyen modelos a seguir ni son universales, sino que son por el contrario localizadas y se configuran socialmente. Una comunidad entendida como los territorios, las personas, las instituciones, las costumbres y las prácticas, se organiza y da sentido al construir una experiencia común y así unos valores, significados y prioridades comunes. En la medida en que un reparto de lo sensible determina qué es visible, determina por consiguiente qué no es visible, ni escuchable, ni es objeto de una experiencia posible.

Aquello que se concibe como 'arte' dentro del reparto de lo sensible, depende de las maneras en que se lo identifica y define, según se entiende qué es lo propio de una comunidad, lo común de lo compartido. En otras palabras: qué se considera arte, cuál es su finalidad (si la tiene), cuáles son sus medios y sus objetivos, caen dentro del espectro de la experiencia sensible del mundo compartida por una comunidad. Desde este punto de vista, un cierto reparto de lo sensible puede llegar a concebir el arte como aquello que debe representar con mayor exactitud y verosimilitud algo (un objeto, una divinidad, una situación, una persona); o como algo que es utilizado para educar y perpetuar cierto tipo de organización de la sociedad. A estas dos formas las llama Rancière el régimen representativo y ético del arte respectivamente (2009, pp. 20-25). Los regímenes son formas en que los espacios, lugares y prácticas de los sujetos son entendidos y distribuidos.

Si bien pueden haber momentos y situaciones donde el arte, al cumplir una función de educación moral de los ciudadanos, sea de fundamental importancia para una comunidad, es importante advertir el peligro de la instrumentalización del arte y de las prácticas culturales que puede derivarse de esta concepción. Decir que el arte y las prácticas culturales sólo son legítimas y, en ese sentido, financiables institucionalmente y aceptables socialmente, si cumplen con una función moral, cierra el paso a otro tipo de expresiones y prácticas que, si bien no se adhieren directamente a estos fines, pueden potenciar otras facultades humanas como la

Aquí sensible se entiende en términos sensoriales y materiales (Rancière, 2009, p.9). 
imaginación, la creación, el reconocimiento de la diversidad y el pensamiento crítico.

Esta crítica de Rancière es particularmente pertinente para nuestro contexto: deslegitimar una práctica artística porque no cumple con las expectativas sociales y políticas de 'hablar' del conflicto, de reparar, de cumplir una función terapéutica, o de contribuir a la construcción de memoria, aminora las potencias del arte de cuestionar narrativas y situaciones conflictivas que se encuentran en la base de nuestra construcción como comunidad. Estas consideraciones también tienen efectos, desde lo institucional, que pueden ser perniciosos para el desarrollo de las prácticas artísticas contemporáneas: que se financie o no tal o cual proyecto artístico con base en la forma en que aborda (o no) explícitamente el tema del conflicto, es un camino que las instituciones públicas culturales deben transitar con cuidado.

Dentro de este contexto, lo estético en Rancière hace referencia no a una disciplina de la filosofía o de la teoría del arte, sino por el contrario a uno de esos regímenes de visibilidad del arte, dentro del cual el arte mismo traba una relación fundamental con aquellas acciones y configuraciones propiamente políticas de una sociedad: el régimen estético. Este régimen estético es especialmente pertinente cuando intentamos abordar las potencias del arte en una época de transición política.

Ahora bien, cuando hablamos de una relación estética entre arte y política, no hablamos de una identificación del arte con un objetivo o filiación política particular, ni de una 'estetización' de la política. La relación estética se basa en que la experiencia sensible, compartida, es disputable. Para Rancière esto significa que, si bien hay un orden establecido de las cosas, las artes, sea bajo un alejamiento del mundo de la vida (como lo intentarían algunos movimientos como el modernismo o el arte abstracto), sea bajo una fusión directa con el mundo de la vida (como lo intentaría el arte relacional), tienen la potencia de cuestionar el orden establecido de los lugares, los espacios, las funciones y los significados que se juegan en lo común. La potencia estética del arte sucede entonces en la introducción de una fractura, de un disenso, que desarma las formas cotidianas y establecidas de estar en el mundo.

Este cuestionamiento, este rompimiento de los consensos sociales sobre lo que es visible y experimentable, constituye lo propiamente político para Rancière. Nos dice el francés:
La política consiste en reconfigurar el reparto de lo sensible que define lo común de la comunidad, en introducir sujetos y objetos nuevos, en volver visible aquello que no lo era y hacer que sean entendidos como hablantes aquellos que no eran percibidos más que como animales ruidosos. (Rancière, 2012, p. 35)

Es precisamente en los momentos de transición como el colombiano, que todas aquellas experiencias, injusticias, violencias y narrativas de diversa índole entran en el campo de lo disputable: el relato oficial de nuestra historia, nuestra subjetividad, nuestras prácticas, entra en cuestión. Asimismo, se abre la posibilidad de repensarnos como comunidad de una forma nueva. Siguiendo a Rancière, podemos plantear que si bien las artes no están obligadas a hacer estos cuestionamientos (no hay un 'deber ser' esencial a las prácticas artísticas), tienen ciertamente la potencia de provocarlos en los sujetos que asisten a un museo, se ven envueltos en un happening, van a una exposición de fotografía, al cine, al teatro, o a un performance.

De igual manera, al aproximarse a las relaciones entre prácticas artísticas y posconflicto, es importante advertir el peligro de una banalización o instrumentalización de la experiencia de la violencia o del trauma en la construcción de una experiencia estética que trate de 'hablar' de la historia del conflicto armado. En este sentido, las reflexiones de Gamboa (2016) son sumamente pertinentes. En su artículo “Víctimas del arte: reflexiones en torno a la representación de la guerra en Colombia", expone los peligros latentes en una apropiación acrítica de la experiencia de la guerra por parte de las prácticas artísticas. Al instrumentalizar esta experiencia, en lugar de producir un reconocimiento crítico del espectador respecto del devenir histórico de su comunidad (en este caso, una comunidad trazada por la guerra) borra su relación con esta neutralizando toda reflexión: "una vez se estereotipa la guerra, se invisibiliza nuestro lugar de enunciación: contemplar el horror de la guerra, manifiesto en el sufrimiento de las víctimas, ayuda a estabilizar nuestro lugar como sujetos" (Gamboa, 2016, p. 36).

En Sobre políticas estéticas (2005) Rancière hace particular énfasis en la potencia estética del arte público y del arte que excede los espacios institucionales del mundo del arte: instalaciones, happenings, performances; pero también obras cinematográficas, fotográficas e intervenciones urbanas. Exceder estos espacios supone, en muchos casos, cambios críticos 


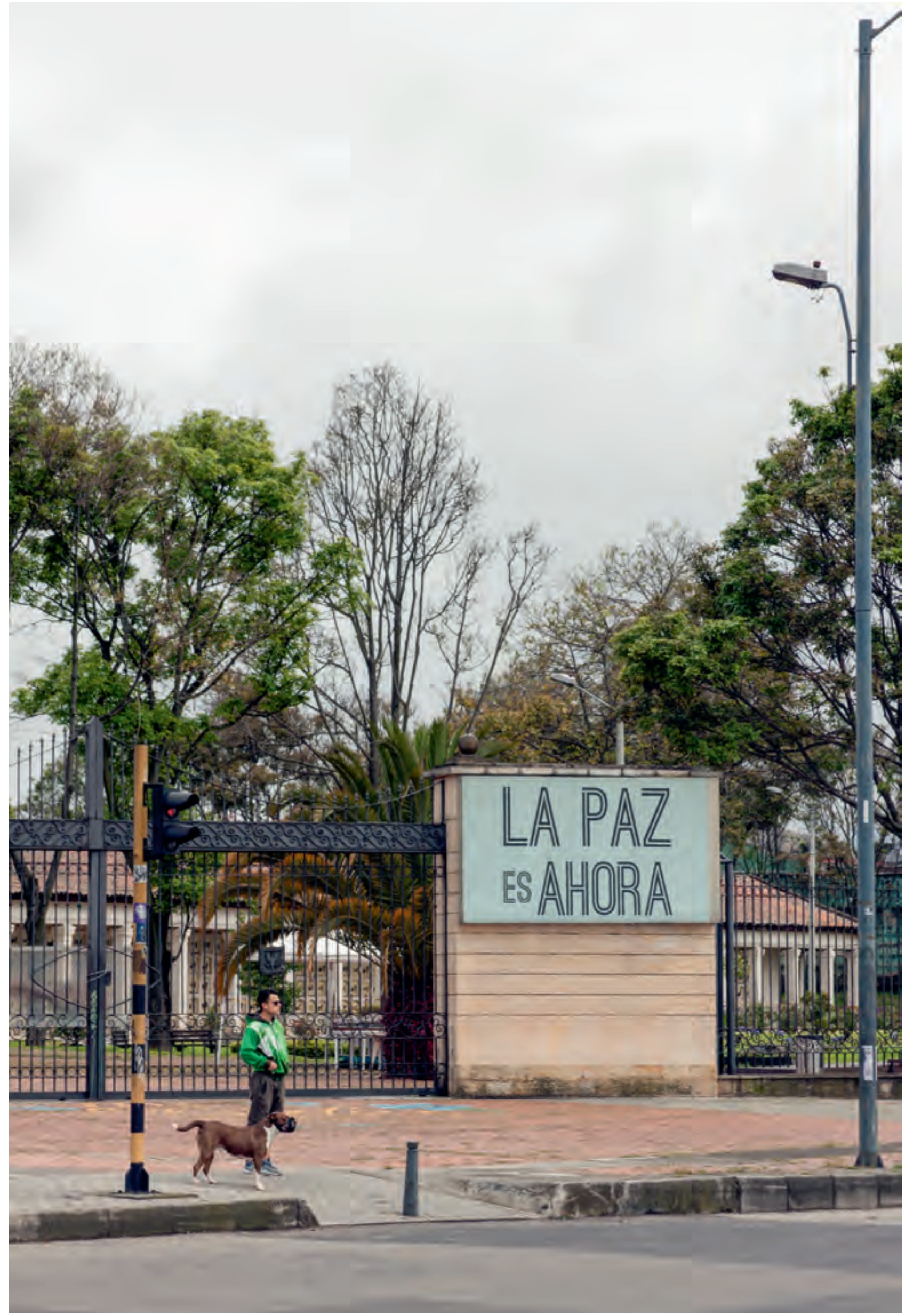

Fotografía: Pedro Pablo Gómez (Bogotá, 2018). Cetro de Memoria, Paz y Reconciliación. 
en la construcción misma de las obras y asume en el encuentro con el incauto y el transeúnte una posibilidad de transformación, rechazo o aceptación, que no puede ser planeada de antemano. Pero, sobre todo, asume que debe mezclarse y abrirse a otras disciplinas y miradas por fuera del mundo del arte, como la arquitectura o el diseño. En el contexto de transición y posconflicto es pertinente también plantear esas mezclas con ciencias sociales y humanas, que pueden proveer un armazón conceptual propio para abordar esta coyuntura.

De alguna forma, buscamos un arte con la potencia de generar una reflexión crítica sobre la relación del espectador con la guerra, con la violencia (directa y simbólica) que hace parte de su historia, de todo aquello que hace parte de sí, pero hasta entonces le era invisible. Que no borre, como denuncia Gamboa (2016), nuestro lugar en el conflicto.

\section{Disenso y arte público}

Ahora bien, una de las preguntas fundamentales que surge de estas consideraciones es la de cómo conjugar esta potencia inherente de las prácticas artísticas con las condiciones y límites institucionales que un contexto de posconflicto nos impone, en cuanto proceso político que atañe de forma fundamental al Estado. Esta pregunta tiene, por lo menos, dos caras. Por un lado, nos podemos preguntar cómo efectivamente se ve un arte público disensual en clave de transición política. Por otro lado, es crucial preguntarnos por el rol de las instituciones públicas que dictaminan las políticas culturales -nacionales, departamentales, locales- en este contexto.

Para abordar la primera pregunta, propongo que enfoquemos nuestra mirada en el concepto de "arte público" como una posible forma en que las prácticas artísticas de la transición pueden materializarse.

En su breve texto An Artist's Statement (2009), la artista estadounidense Andrea Fraser llama la atención sobre la doble interpretación que puede hacerse del término 'arte público'. Podemos entender el arte público como el arte que se exhibe en y por instituciones públicas, o el arte que sale de los espacios usuales de exhibición artística como museos o galerías y se instala en el espacio público, sea o no financiado por instituciones públicas (Fraser, 1992, pp. 326-327). La reflexión de Fraser no pretende defender una práctica artística particular — sea esta de arte clásico, popular, público, en fin-, sino entender de forma crítica el rol del artista en estas prácticas, y cómo incluso el acto deliberado de abandonar un lugar específico —un museo- no necesariamente significa que una práctica artística esté rompiendo con los esquemas clásicos de producción que ella implica. Es decir, que la práctica misma, el cómo, en su abandono de lineamientos y lugares establecidos, debe ser asumida de forma crítica por el artista si la pretensión de su intervención es introducir una transformación en lo real.

Esta reflexión de Fraser nos permite entender que, por un lado, no todo arte promovido, financiado o exhibido por una institución pública es necesariamente acrítico, o carece de potencia crítica. Por otro lado, que por el hecho de salir de los espacios institucionales designados, una práctica artística no comporta necesariamente un carácter crítico o disensual, como se elaboró en la sección anterior según Rancière.

Así, gran parte de la potencia estética y disensual del arte público depende del cómo: puede tratarse de una pieza financiada con fondos públicos, o una pieza que sale al encuentro y se instala en el espacio público. En ambos casos, hablamos de piezas que crean rupturas y abren espacios de novedad cuando su manera, el cómo, es aquello que irrumpe en la cotidianidad y suspende temporalmente la forma habitual en que entendemos el mundo, escuchamos y reconocemos a los otros como parte del mismo reparto de lo sensible. En este proceso, reconoce Fraser, debe implicarse el artista mismo: al ejercer algún tipo de crítica, sea a la institución del arte o a otra realidad, el artista no puede ignorar el papel que él mismo juega como parte de esta institución o realidad, cómo reproduce jerarquías, patrones de violencia simbólica, etcétera.

En este sentido, cuando pensamos en un arte público de la transición, entendemos que la pretensión no es únicamente la de llevar la crítica 'hacia fuera', es decir, 'a la gente', 'a la sociedad', 'al Estado'. En este movimiento, el artista es el primero que se expone como sujeto en transición, en cuestión, en disputa. Se abre al otro y se desdibuja en su propia práctica para permitir una suerte de suspensión de la historia en la que crear otras formas de nombrarnos como sujetos, de reconocernos, y reconocer las injusticias y violencias que hacen parte estructural del tejido social, se muestran como tareas necesarias.

Entender el arte público en transición de esta manera nos obliga a trascender la visión tradicional, común 
en algunas entidades estatales, de concebirlo como la instalación de monumentos o estatuas conmemorativas. Igualmente, el espacio público no es lo que entendemos tradicionalmente, a saber, el espacio topográfico por donde transitan los ciudadanos y cuyo cuidado y conservación está a cargo del Estado. Según Luisa Roa (2015, p.34), los debates más recientes respecto de esta forma de arte, en particular el que sucede en el espacio público, asume que este último es un concepto abierto, que no está completamente cerrado y determinado. En este sentido, el espacio público está en constante transformación, de acuerdo con lo que, desde Rancière, podríamos determinar como configuraciones de reparto de lo sensible. Estas, al estar atadas a la historia y devenir de las comunidades, implican momentos de cambio y transformación. En un contexto de transición, muy posiblemente una intervención de este talante no 'llega a instalarse' en el espacio, sino que crea el espacio mismo en el que se visibilizan y se disputan nombres, relaciones, impugnaciones, que nunca antes habían sido nombradas. La potencia de esta afirmación en el contexto de transición se insinúa: que este es un momento histórico donde los espacios mismos, ya sobre-determinados, pueden reconfigurarse y transformarse de acuerdo a las nuevas relaciones, necesidades y choques que los cambios sociales y culturales de la transición provoquen.

Es fundamental aclarar, en este punto, que la conexión que se ha trazado entre las prácticas artísticas, su potencial estético y su lugar en el posconflicto, y el arte público, en ningún momento pretende argumentar que esta sea la única forma en que esta relación puede materializarse. Si bien lo postulo como una potencia, esto no quiere decir que sea la única configuración posible de esta relación. Por el contrario, considero que, si desde el arte público logra trabarse una relación fértil con la transición política y social que estamos viviendo, es muy posible que a futuro veamos el surgimiento de otras y nuevas formas de abordar esta relación, que incluso reten e impugnen la idea misma de arte público. Esto sería, de hecho, algo completamente deseable.

\section{Arte público y políticas culturales de transición}

Es momento entonces de abordar la segunda pregunta planteada en la sección anterior respecto del rol de las instituciones públicas en nuestro contexto. La pregunta desde lo público en cuanto Estado, es clara: ¿cómo formular unas políticas culturales que se adapten a las exigencias y necesidades de la transición y el posconflicto? Sobre todo, ¿cómo hacerlo sin zanjar las políticas ya existentes que no tienen una relación directa o implícita con el posconflicto? ¿Cómo promover un arte público, en sus diversas acepciones, que permita esos espacios de novedad, sin que se convierta en un instrumento estatal de promoción de la historia oficial, establecida, predeterminada?

Según Roa (2015), “La importancia de las acciones es encontrar puntos de tensión en la esfera pública, con el fin de recordar y asomarse a un sistema de relaciones que parecen invisibles pero que rigen la forma en que se organiza un lugar" (37). Esta reflexión es valiosa en la medida en que permite poner el debate en perspectiva. En primer lugar, no se trata de que haya o no haya políticas culturales para la transición sin más, sino de entender cuál sería su lugar, cuáles rupturas y tensiones propone, y cuáles son los peligros que deben advertirse al formularlas. En segundo lugar, llama nuestra atención sobre el hecho de que esta potencia estética del arte sucede de forma local y localizada. Es decir, no podemos predeterminar las formas ni lugares específicos en que estas prácticas se materializan, pero podemos velar porque su potencia creativa, disensual, no sea suprimida o neutralizada.

De hecho, en la medida en que van a surgir nuevos debates, nuevas iniciativas y necesidades de las comunidades artísticas y en general, es pertinente afirmar que se necesitan unas políticas culturales para la transición y el posconflicto que atiendan las complejidades y especificidades de estas etapas. Es importante que el Estado - a nivel nacional y localtenga unas líneas y lineamientos claros de inversión de recursos según los requerimientos artísticos y culturales de participación en este proceso. Asegurar una financiación, un recurso material explícito para este tipo de intervenciones es también garantizar la participación directa de la comunidad artística en un proceso de transformación y estabilización social crucial para el futuro del país. En este caso, no se trata, quizás, de una posición sobre lo que son o no las prácticas artísticas, sino de lograr que tengan un lugar simbólico, físico y material, en el proceso de reconstrucción social y de transformación histórica. Nuevamente, es importante aclarar que estas políticas no deben explicitar las formas concretas que estas prácticas e intervenciones deben tener, so pena de contravenir el objetivo mismo del tipo de participación que aquí se plantea. Se trata de que el Estado reconozca y garantice un recurso material (al arte público 
como arte financiado por instituciones públicas), al igual que un espacio físico para expresiones alternativas y transgresoras que interpelen, desde su diversidad, nuestro imaginario de comunidad (el arte público como arte que se instala en el espacio público).

En este punto nos encontramos con la ambivalencia propia del arte público en sus dos acepciones: sus potencias y sus peligros. En palabras de Jane Rendell (2015), el aspecto clave del arte público es "la necesidad de expresar ambiguamente, de ofrecer una cantidad de interpretaciones que más que decirnos qué debemos pensar, nos pide cuestionar la manera en que le asignamos valor a las cosas" (p. 75). Así, por un lado, tenemos la potencia de la pregunta, que abre nuevas posibilidades de producción de sentido sobre lo común. Por otro lado, citando al teórico cultural Malcolm Miles, se advierte que el gran peligro de este tipo de arte es "su uso como papel tapiz para cubrir los conflictos sociales y su uso como monumento para promover las aspiraciones de sus patrocinadores corporativos" (Rendell, 2015, p. 72). Tener en cuenta esta advertencia es crucial en el momento de formular unas políticas culturales que efectivamente atiendan al carácter creativo y estético del arte, sin instrumentalizarlo con agendas políticas específicas. En este sentido, Bustos (2017) ha hecho un trabajo extenso de análisis donde muestra que la 'cultura' siempre ha sido entendida, desde el Estado, como un instrumento o un lugar para promover la paz y la convivencia. Su análisis de esta relación y su naturalización desde lo público apunta a la necesidad del sector cultura de re-pensar su lugar y sus estrategias en un contexto amplio de posconflicto como el actual.

El asunto de cuidado en este debate es lograr que, al formular unas políticas culturales para la transición y el posconflicto, los programas y estímulos que existen actualmente y que han recorrido un largo camino para su consolidación, no se vean afectados por esto. German Rey (2017) lleva a cabo un detallado análisis de la evolución de las políticas culturales en Colombia desde tres ámbitos: la lectura, el cine y la reciente incorporación de la economía naranja. En su artículo podemos ver cómo las políticas culturales desde ámbitos como la lectura han tenido un desarrollo paulatino casi desde la creación de la Biblioteca Nacional, mientras que otros ámbitos apenas están empezando a abrirse campo, como es el caso de la llamada economía naranja. Rey destaca la necesidad de que las políticas culturales estén atadas al devenir de lo social y reconozcan las necesidades actuales, reconozcan los retos que conciernen a su impacto a largo plazo, y que atiendan de forma diferencial las regiones. Esto se vuelve aún más urgente en un contexto de posconflicto.

Otra exigencia que nuestro contexto político impone es que las instituciones culturales flexibilicen las ideas que tienen sobre cómo las prácticas artísticas pueden involucrarse con la situación política del país, de manera tal que no se vean inmersas en una lógica de reproducción de los discursos y narrativas oficiales. Una intervención en el espacio público puede no hablar explícitamente del conflicto armado, pero sí puede llamar la atención de los transeúntes sobre las violencias cotidianas, directas y simbólicas, que la guerra ha normalizado en las formas de reconocimiento y relacionamiento con el otro.

A nivel nacional, dos instrumentos de política cultural cobran relevancia. En primer lugar, la Cátedra para la cultura de paz desde la diversidad y la creatividad realizada en 1999, evento de reflexión teórica y práctica sobre la cultura de paz y la convivencia desde las prácticas artísticas y culturales. Los objetivos de este evento son:

- Despertar la conciencia de la opinión pública nacional e internacional sobre el tema del patrimonio cultural como espacio de reflexión y tolerancia.

- Alertar a la comunidad cultural y a los actores del conflicto sobre las consecuencias que sufre nuestro patrimonio por causa de la confrontación.

- Reactivar y poner en acción recursos y programas orientados al desarrollo productivo del patrimonio cultural. (Ministerio de Cultura, 1999, p. 7)

Este documento está enfocado en asuntos patrimoniales, de cuidado y conservación, y de las relaciones de las prácticas artísticas y culturales con temas de desarrollo, medio ambiente, patrimonio y diversidad cultural.

En segundo lugar, en 2001 el Ministerio de Cultura emitió el Plan Nacional de Cultura con vigencia de diez años, cuya apuesta principal está "dirigida hacia la construcción de una ciudadanía democrática cultural" (Ministerio de Cultura, 2001, p. 9). Asume como eje la idea de una ciudadanía plural y diversa que tienda hacia una convivencia democrática e inclusiva. En 2011 este plan fue ratificado y continúa siendo el instrumento, desde el Ministerio de Cultura, para entender y ejecutar la política cultural del país.

Estos dos documentos son instrumentos valiosos en términos de una política cultural a nivel nacional. 


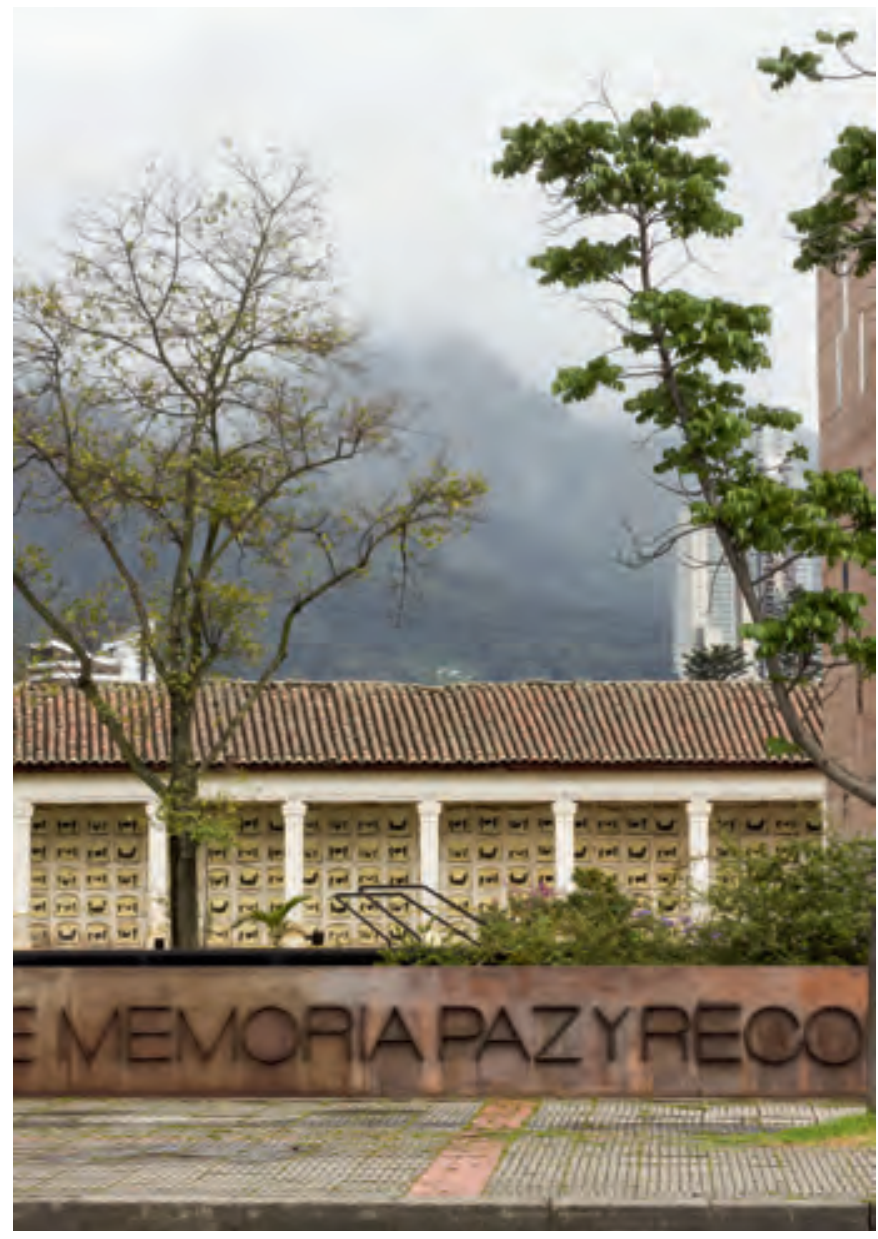

Fotografía: Pedro Pablo Gómez (Bogotá, 2018). Cetro de Memoria, Paz y Reconciliación.

Sin embargo, la temporalidad de los documentos y la coyuntura histórica actual exigen, si no un cambio, por lo menos actualizaciones importantes, en particular del Plan Nacional de Cultura. Esta reevaluación de los instrumentos de política respondería no solo al contexto de tránsito y posconflicto, sino también a las transformaciones de los campos artísticos y culturales que el Plan mismo ha fomentado. De igual manera, vale la pena plantear el debate sobre los instrumentos de medición y los indicadores que permiten ver los impactos de las políticas, aun cuando esta medición sea siempre parcial. Este debate implicaría una revisión no sólo de los indicadores como tal, sino de las formas en que la información es recogida e interpretada 5 . Una revisión tal nos obligaría a plantear la discusión entre la visión estatal de las políticas culturales y las iniciativas y -especialmente- las críticas que surgen desde

5 Sobre esto, es importante tener en cuenta debates sobre lo cultural y los comunes o commons, como forma de repensar las políticas culturales y los instrumentos de medición correspondientes (ver Barbieri, 2014; 2015). espacios no estatales en relación con ella. En este caso, nos veríamos en la necesidad de trazar nuevas formas de comunicación y participación entre el Estado y los actores, para que estas políticas sean sensibles a los cambios y necesidades propias del contexto.

Finalmente, una política cultural que atienda a las particularidades del contexto actual, que intenta pensar el arte público (entre otros) desde una potencia estética y disensual, sería también una política transitoria. Los retos institucionales del posconflicto exigen al Estado formular políticas y maneras de hacer que se adapten a la transición, que permitan, en efecto, que la transición sea exitosa y que una vez realizada, de lugar a la formulación de unas nuevas políticas para un país que ha superado el conflicto armado como parte de la esfera pública y de la cotidianidad de sus ciudadanos. En este sentido, advierte Rancière:

Hay dos clases de espacios del arte: las instituciones oficiales con sus programas, y los espacios «ordinarios», los espacios combinados donde la «educación estética» funciona a través de la indistinción misma entre diversión y arte. Y nos recuerda también que las instituciones mismas son lugares de tránsito ${ }^{6}$, lugares aleatorios de encuentro con lo heterogéneo, que facilitan procesos concretos de reconfiguración de las identidades y de los campos de experiencia. (2005, p. 69).

Es pues crucial pensar en unas políticas culturales para la transición que sean, a su vez, transitorias, teniendo en cuenta que las prácticas artísticas en este contexto tienen la potencia de propiciar nuevos espacios de encuentro, debate, impugnación y creación de símbolos y narrativas de una comunidad que se redefine a sí misma, para proyectarse a un futuro distinto. En este sentido, la creación artística como disenso, como introducción de lo nuevo y de "quienes no tienen parte", impone la necesidad de pensarnos como miembros de una comunidad radicalmente diversa: que el conflicto entre formas de vida y prácticas culturales está en el origen de nuestra comunidad y que no deben eliminarse. Una comunidad estética del disenso es una comunidad siempre en movimiento, re-definiendo las fronteras de aquellos objetos y experiencias que hacen o no parte de eso común, de los sujetos y las prácticas que son visibles y que no.

6 El énfasis es mío. 
Cuando el arte interviene en ese reparto de lo sensible establecido, cuando irrumpe suspendiendo las maneras habituales en que se entiende la experiencia cotidiana, el mundo y los lugares que los sujetos ocupan en una sociedad, se da igualmente para Rancière una suspensión de las formas de dominación que han sido naturalizadas. De la razón sobre lo sensible, del Estado sobre la sociedad, de la humanidad sobre la naturaleza. Entender el disenso y la diferencia como parte fundamental de nuestro tejido social, herido y maltrecho, es un paso fundamental hacia la construcción de una sociedad que transita a una nueva etapa en su historia, que no homogeniza el pensamiento ni la vida, sino que permite que esos disensos y diferencias convivan y enriquezcan la vida en común.

\section{Referencias}

Acuerdo final para la terminación del conflicto y la construcción de una paz estable y duradera (Noviembre 24 de 2016) [en línea].Disponible en: http://www.altocomisionadoparalapaz.gov.co/mesadeconversaciones/ PDF/24-1480106030.11-1480106030.2016nuevoacuerd ofinal-1480106030.pdf

Barbieri, N. (2015) Bibliotecas públicas: entre la medición de su impacto y la evaluación de su valor público. En Ministerio de Educación, Cultura y Deporte, VII Congreso Nacional de Bibliotecas Públicas, pp. 83-87 [en línea]. Disponible en: http://ddd.uab.cat/ record/163522/

(2014). Cultura, políticas públicas y bienes

comunes: hacia unas políticas de lo cultural. En Ágora, 1(1), pp. 101-119. doi: http://dx.doi.org/10.6035/ Kult-ur.2014.1.1.3

Bustos, M. L. (2017). Las políticas culturales y la construcción de paz. En Bustos M. L., Peñuela J. y Romero S., Cruce de caminos (pp. 13-54). Bogotá: Universidad Distrital Francisco José de Caldas.

Fraser, A. (2009). An Artist's Statement (1992). En Alberro, A. y Stimson, B. (Eds.), Institutional Critique. An Anthology of Artist's Writings (pp. 318-329). Cambridge/London: The MIT Press.

Fischer, M. (2011). Transitional Justice and Reconciliation: Theory and Practice. En Austin, B., Fischer, M. y Giessmann H. J. (eds.), Advancing Conflict Transformation. The Berghof Handbook II (pp. 405430). Opladen/Framington Hills: Barbara Budrich
Publishers [en línea] Disponible en www.berghof-handbook.net.

Gamboa, A. (2016). Víctimas del arte: reflexiones en torno a la representación de la guerra en Colombia. En Calle 14 revista de investigación en el campo del arte, 11 (19), pp. 30-43. doi: https://doi.org/10.14483/udistrital. jour.c14.2016.2.a03

GMH (2013). iBasta ya! Colombia: Memorias de guerra y dignidad. Bogotá: Imprenta Nacional.

Ley 975 de 2005 (Julio 25). Por la cual se dictan disposiciones para la reincorporación de miembros de grupos armados organizados al margen de la ley, que contribuyan de manera efectiva a la consecución de la paz nacional y se dictan otras disposiciones para acuerdos humanitarios.

Miall, H. (2004). Conflict Transformation: A MultiDimensional Task. En Austin A., Fischer, M. y Ropers N. (eds.), Transforming Ethnopolitical Conflict (pp. 67-90). Wiesbaden: The Berghof Handbook [en línea]. Disponible en: www.berghof-handbook.net.

Ministerio de Cultura (1999). Cátedra para la cultura de la paz. Desde la diversidad y la creatividad. Bogotá: Ministerio de Cultura.

(2001). Plan nacional de cultura. Bogotá:

Ministerio de Cultura [en línea]. Disponible en: http:// www.mincultura.gov.co/planes-y-programas/Planes/ plan\%20nacional\%20de\%20cultura/Paginas/default. aspx

Nasi, C. y Rettberg, A. (2005). Los estudios sobre conflicto armado y paz: un campo en evolución permanente. En Colombia Internacional 62, jul-dic 2005, pp. 64-85.

Rancière, J. (2012). El malestar en la estética. Buenos Aires: Clave Intelectual, S. L.

(2009). El reparto de lo sensible. Santiago de Chile: LOM Ediciones.

(2005). Sobre políticas estéticas. Barcelona: Museu d'Art Contemporani de Barcelona y Servei de Publicacions de la Universitat Autónoma de Barcelona.

Rendell, J. (2015). From Critical Spatial Practice to SiteWriting. En Holub, B. y Hohenbüchler, C. (Eds.), Planning 
Unplanned - Towards a New Positioning of Art in the Context of Urban Development (pp. 71-84). Austria: Verlag für Moderne Kunst.

Rey, G. (2017). Del dicho al hecho. El trecho recorrido por algunas políticas culturales colombianas. En Calle 14 revista de investigación en el campo del arte, 12(22), pp. 174-187. doi: https://doi.org/10.14483/21450706.12352

Roa, L. (2015). Acerca del Arte en Espacio Público. En Alcaldía Mayor de Bogotá, Secretaria de Cultura, Recreación y Deporte, Arte en espacio público, pp. 33-39.

Traba, M. (1974). La cultura de la resistencia. En: Revista de Estudios Sociales No. 34 diciembre de 2009, pp. 136-145.
USIP (2009). Guiding Principles for Stabilization and Reconstruction. Washington D. C.: United States Institute of Peace and U.S. Army Peacekeeping and Stability Operations Institute (PKSOI).

Webel, C. y Galtung, J. (Eds.). (2007). Handbook of Peace and Conflict Studies. New York: Routledge, Taylor \& Francis e-Library.

Zuleta, E. (2004). Sobre la Guerra. En Elogio de la dificultad y otros ensayos (pp. 71-74). Cali: Fundación Estanislao Zuleta. 